\title{
Magnetic and crystal structures of the one-dimensional ferromagnetic chain pyroxene $\mathrm{NaCrGe}_{2} \mathrm{O}_{6}$
}

\author{
G. Nénert, ${ }^{1, *}$ C. Ritter, ${ }^{1}$ M. Isobe, ${ }^{2}$ O. Isnard, ${ }^{3}$ A. N. Vasiliev, ${ }^{4}$ and Y. Ueda ${ }^{2}$ \\ ${ }^{1}$ Institut Laue-Langevin, Boîte Postale 156, 38042 Grenoble Cedex 9, France \\ ${ }^{2}$ Institute for Solid State Physics, University of Tokyo, 5-1-5 Kashiwa, Chiba 277-8581, Japan \\ ${ }^{3}$ Institut Néel, CNRS/Université Joseph Fourier, BP 166, 38042 Grenoble, France \\ ${ }^{4}$ Department of Low Temperature Physics, Moscow State University, Moscow 119991, Russia \\ (Received 4 February 2009; revised manuscript received 14 April 2009; published 1 July 2009)
}

\begin{abstract}
We investigated the magnetic and structural properties of the ferromagnetic pyroxene $\mathrm{NaCrGe}_{2} \mathrm{O}_{6}$ by superconducting quantum interference device magnetometry and powder neutron diffraction. This material is the only ferromagnetic member of the recently reported multiferroic pyroxene family $A M \mathrm{X}_{2} \mathrm{O}_{6}(A=\mathrm{Li}, \mathrm{Na} ; M$ $=\mathrm{Fe}, \mathrm{Cr} ; X=\mathrm{Si}, \mathrm{Ge})$. Below $T_{C}=6 \mathrm{~K}$, the magnetic structure is characterized by one-dimensional magnetic chains with spins aligned along the $c$ axis of the monoclinic cell. The magnetic moment of $\mathrm{Cr}^{3+}$ is significantly reduced by about $25 \%$. We show that this is likely the result of the low dimensionality of the system. The associated magnetic space group is $C 2^{\prime} / c^{\prime}$. This symmetry does not allow a linear magnetoelectric effect. No structural phase transition was observed down to $1.8 \mathrm{~K}$.
\end{abstract}

DOI: 10.1103/PhysRevB.80.024402

PACS number(s): 75.25.+z, 91.60.Pn, 75.50.Ee

\section{INTRODUCTION}

In recent years, the coupling between magnetic and dielectric properties in transition-metal oxides gave rise to significant research effort. ${ }^{1-3}$ This effort is governed by the emergence of new fundamental physics and potential technological applications. ${ }^{2-4}$ Multiferroic materials exhibit simultaneously (ferro)magnetic, pyroelectric, and ferroelastic properties. Contrary to multiferroic materials, magnetoelectric materials show an induced electrical polarization by a magnetic field. A proper understanding of the interplay between the various physical properties of these two types of materials relies heavily on the knowledge of the detailed crystal and magnetic structures.

Recently pyroxene materials with the general formula $A M \mathrm{Si}_{2} \mathrm{O}_{6}(A=\mathrm{Li}, \mathrm{Na} ; M=\mathrm{Fe}, \mathrm{Cr})$ have been reported as multiferroic materials. ${ }^{5}$ This family of materials provides a large playground for physicists in condensed matter where $A$ may be alkali or alkaline-earth elements, $M$ may be various metals with valence state $2+$ or $3+$, and $\mathrm{Si}^{4+}$ can be replaced by $\mathrm{Ge}^{4+}$. Due to the presence of chains of octahedra that can be magnetic, this family of materials has attracted much attention. It exhibits interesting properties such as the spin gap system found in $\mathrm{NaTiSi}_{2} \mathrm{O}_{6}$ (Ref. 6) or the low-dimensional magnetism found in $\mathrm{LiVGe}_{2} \mathrm{O}_{6}{ }^{7}$

While these pyroxenes have been the subject of various studies, ${ }^{5-12}$ the complete magnetic structures are known only for few members of this class. ${ }^{7,11-15}$ We have started recently to investigate the magnetic structure of several pyroxene materials. ${ }^{14-16}$ The investigation of this magnetically frustrated family by neutron diffraction is of prime importance in the interpretation and/or prediction of their multiferroic/ magnetoelectric properties.

Jodlauk et al. ${ }^{5}$ suggested that several members of the pyroxene family should have an incommensurate magnetic structure. This suggestion was based on the geometrical magnetic frustration present in this family and some theoretical assumptions. ${ }^{5,17}$ However it turns out for the moment that most of the pyroxenes exhibit a simple magnetic struc- ture commensurate with the lattice with $\mathbf{k}=\mathbf{0} .^{7,11,12,14,15} \mathrm{Nev}-$ ertheless, several of those reported magnetic structures are compatible with a linear magnetoelectric effect. ${ }^{7,14,15}$

In this contribution, we continue our investigation of the crystal and magnetic structures of the pyroxene family with its ferromagnetic member: $\mathrm{NaCrGe}_{2} \mathrm{O}_{6}$. We have investigated its magnetic properties using superconducting quantum interference device (SQUID) magnetometry and powder neutron diffraction as function of temperature. We show that $\mathrm{NaCrGe}_{2} \mathrm{O}_{6}$ exhibits like $\mathrm{Li} M \mathrm{Si}_{2} \mathrm{O}_{6}(M=\mathrm{V}, \mathrm{Cr}, \mathrm{Fe})$ a commensurate magnetic structure characterized by $\mathbf{k}=\mathbf{0}$. ${ }^{7,11,14}$ The associated magnetic symmetry is $C 2^{\prime} / c^{\prime}$ and does not allow a linear magnetoelectric effect. No structural phase transition was observed down to $1.8 \mathrm{~K}$. We find that the magnetic moment of $\mathrm{Cr}^{3+}$ is significantly reduced by about $25 \%$. This effect is interpreted as the result from the onedimensional (1D) crystal structure of the system. Magnetic susceptibility data are well described by a 1D Heisenberg chain of $S=3 / 2$ spins.

\section{EXPERIMENT}

Polycrystalline samples of $\mathrm{NaCrGe}_{2} \mathrm{O}_{6}$ were prepared by a solid-state reaction with an appropriate molar ratio of $\mathrm{Na}_{2} \mathrm{CO}_{3}, \mathrm{Cr}_{2} \mathrm{O}_{3}$, and $\mathrm{GeO}_{2}$. The weighted mixtures were pressed into pellets and heated at $1273 \mathrm{~K}$ in air for several days with one intermediate grinding.

Polycrystalline $\mathrm{NaCrGe}_{2} \mathrm{O}_{6}$ magnetization measurements were carried out by a superconducting quantum interference device magnetometer in the temperature range of $2-350 \mathrm{~K}$ and external magnetic fields of 50, 1000, and 50000 Oe.

Neutron-diffraction measurements were carried out on powder $\mathrm{NaCrGe}_{2} \mathrm{O}_{6}$. The data were collected with the double-axis multicounter high-flux diffractometer D1B at the Institut Laue-Langevin ILL Grenoble using $2.52 \AA$ wavelength selected by a pyrolitic graphite monochromator. In the configuration used, the resolution of $\mathrm{D} 1 \mathrm{~B}$ was about full width at half maximum $(\mathrm{FWHM}) \sim 0.3^{\circ}$. The multicounter is composed of 400 cells covering a total angular domain at $80^{\circ}$ 


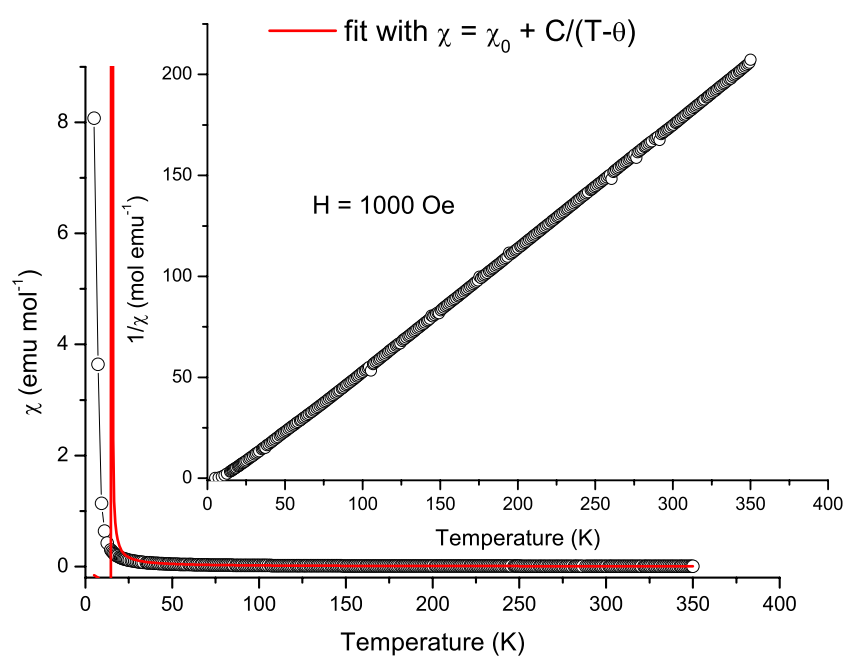

FIG. 1. (Color online) Magnetic susceptibility measured on a $\mathrm{NaCrGe}_{2} \mathrm{O}_{6}$ powder sample. The inset shows the inverse magnetic susceptibility. The line represents a Curie-Weiss temperature dependence fit defined by $\chi=\chi_{0}+\frac{C}{T-\theta}$.


investigated using high-resolution powder data at room temperature, 1.7 and $10 \mathrm{~K}$ from D2B diffractometer at the ILL. The measurements were carried out at a wavelength of $1.594 \AA$ corresponding to the (335) Bragg reflection of a germanium monochromator. The neutron detection is performed with ${ }^{3} \mathrm{He}$ counting tubes spaced at $1.25^{\circ}$ intervals for D2B. A complete diffraction pattern is obtained after about 25 steps at $0.05^{\circ}$ in $2 \theta$. Further investigation of the crystal structure temperature dependence was made using the highresolution D1A diffractometer at the ILL. In the configuration used, the resolution FWHM of D1A was about $0.3^{\circ}$ at $90^{\circ}$. The measurements were carried out at a wavelength of $\lambda=1.909 \AA$ selected by the (115) reflection of a germanium monochromator. The neutron detection was performed with a set of $6^{\circ}$ spaced ${ }^{3} \mathrm{He}$ counting tubes. The complete diffraction pattern was obtained by scanning over the whole $2 \theta$ range. Diffraction data analysis was done using the FULLPROF refinement package. ${ }^{18}$

\section{RESULTS}

\section{A. Magnetic properties}

The measured temperature dependences of the magnetic susceptibility in an external magnetic field of 50 and 1000 Oe are shown in Figs. 1 and 2. From Fig. 2, we see that a ferromagnetic order appears below $T_{C}=6 \mathrm{~K}$. The coercitive field is lower than 1000 Oe.

We present in Fig. 1 the temperature dependence of the magnetic susceptibility and its inverse measured on a powder sample with $H=1000$ Oe. We fitted the magnetic susceptibility with a Curie-Weiss temperature dependence defined by $\chi=\chi_{0}+\frac{C}{T-\theta}$. The fit was made in the range of $150-350 \mathrm{~K}$. We see that the magnetic susceptibility departs from the CurieWeiss model below $25 \mathrm{~K}$. The determined effective moment is $\mu_{\text {eff }}=3.600(9) \mu_{B}$ and $\theta=15.2(5) \mathrm{K}$. This is in agreement

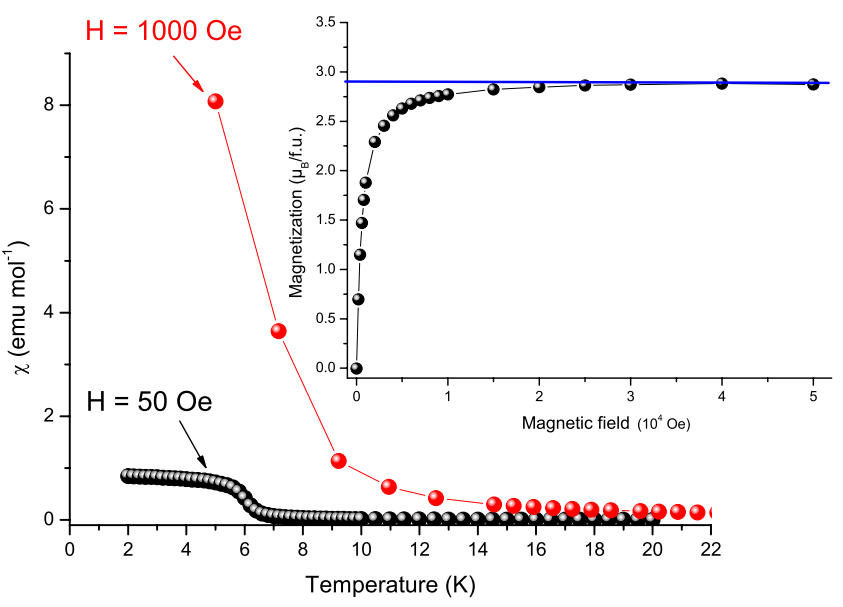

FIG. 2. (Color online) Magnetic field dependence of the magnetization measured on a $\mathrm{NaCrGe}_{2} \mathrm{O}_{6}$ powder sample. The inset shows the magnetic field dependence of the magnetization measured on a $\mathrm{NaCrGe}_{2} \mathrm{O}_{6}$ powder sample.

with a previous report. ${ }^{19}$ The field dependence of the magnetization is presented in the inset of Fig. 2. The saturation value is about $2.9 \mu_{B}$ in a magnetic field of 50000 Oe.

One characteristic of these pyroxene materials is that they are good representative of low-dimensional magnetism with linear chains running along the $c$ axis. ${ }^{19}$ Contrary to the pyroxene crystallizing in the $P 2_{1} / c$ space group, $\mathrm{NaCrGe}_{2} \mathrm{O}_{6}$ is a good representative of one-dimensional magnetic triangular lattice with only two magnetic exchange constants instead of three like in $\mathrm{LiCrSi}_{2} \mathrm{O}_{6}{ }^{5,14}$ Consequently one can try to treat the magnetic susceptibility data using low-dimensional magnetism formula. ${ }^{20}$

For a uniform chain of classical spins based on the Hamiltonian $\mathcal{H}=-2 J \sum_{i} \mathbf{S}_{i} \mathbf{S}_{i+1}-g \mu_{B}[S(S+1)]^{1 / 2} \sum_{i} \mathbf{H} \cdot S_{i}$, the magnetic susceptibility can be expressed as ${ }^{20}$

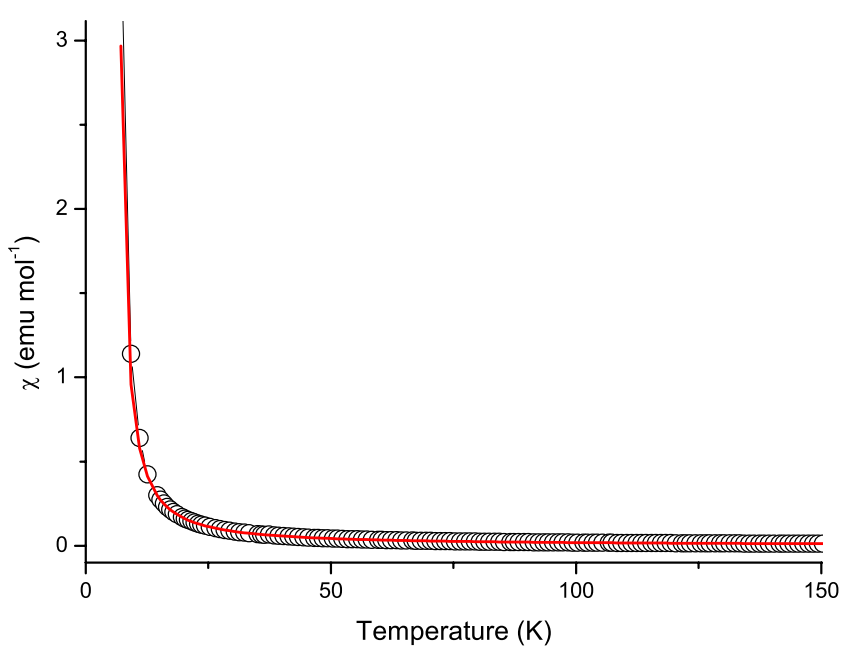

FIG. 3. (Color online) Temperature dependence of the magnetic susceptibility $\chi$. The solid line represents the best fit of the experimental data to Eq. (2) for $T>15 \mathrm{~K}$. 


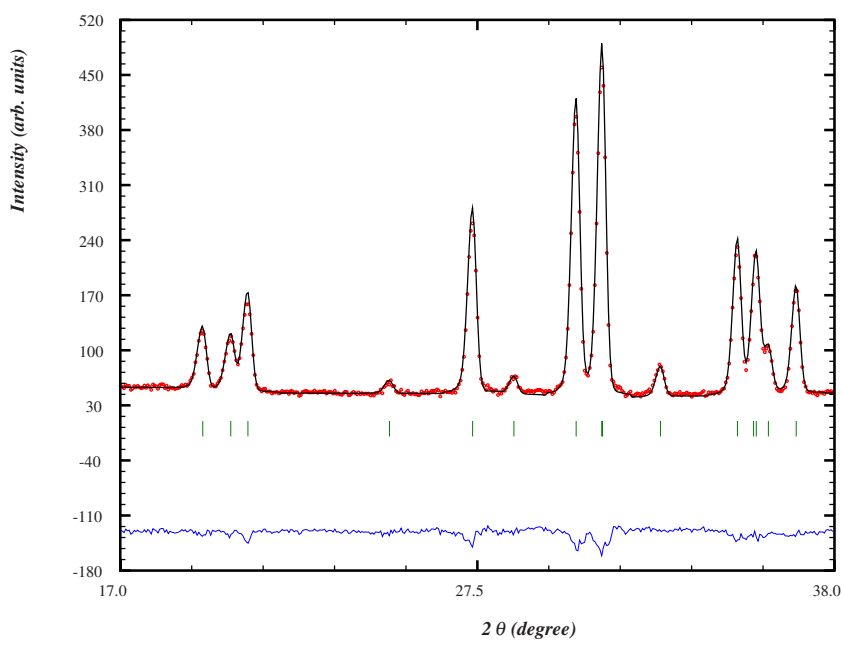

FIG. 4. (Color online) Low angle part of the neutron pattern $(\lambda=1.594 \AA)$ of $\mathrm{NaCrGe}_{2} \mathrm{O}_{6}$ sample collected at room temperature using the D2B diffractometer. The refinement has been done in the $C 2 / c$ space group with the following statistics: $R_{p}=5.07 \%$ and $R_{w p}=6.4 \%$.

$$
\chi_{\text {chain }}=\frac{N g^{2} \beta^{2} S(S+1)}{3 k_{b} T} \times \frac{1+u}{1-u},
$$

where $u$ is the well-known Langevin function defined as $u$ $=\operatorname{coth}\left[2 J S(S+1) / k_{b} T\right]-k_{b} T /[2 J S(S+1)]$ with $S=3 / 2$. Considering the three-dimensional $(3 \mathrm{D})$ ordering at $6 \mathrm{~K}$, we assumed an interchain interaction $J^{\prime}$ between the ferromagnetic chain. Applying the mean-field approximation, the susceptibility of $\mathrm{NaCrGe}_{2} \mathrm{O}_{6}$ can be expressed as

$$
\chi=\frac{\chi_{\text {chain }}}{\left(1-\frac{z J^{\prime}}{N g^{2} \beta^{2}}\right) \chi_{\text {chain }}},
$$

where $z$ is the number of nearest-neighbor chains, $N$ is Avogadro's number, $g$ the $g$ factor of a free-electron spin, and $\beta$ is the Bohr magneton. With $g$ fixed at 2.00 , the leastsquares fit of the experimental data above $15 \mathrm{~K}$ to the above expression led to $J / k_{b}=2.32(1) \mathrm{K}$, a Curie constant $C$ $=\frac{N g^{2} \beta^{2} S(S+1)}{3 k_{b}}=1.666(2)$ emu mol ${ }^{-1} \mathrm{~K}^{-1}$ corresponding to
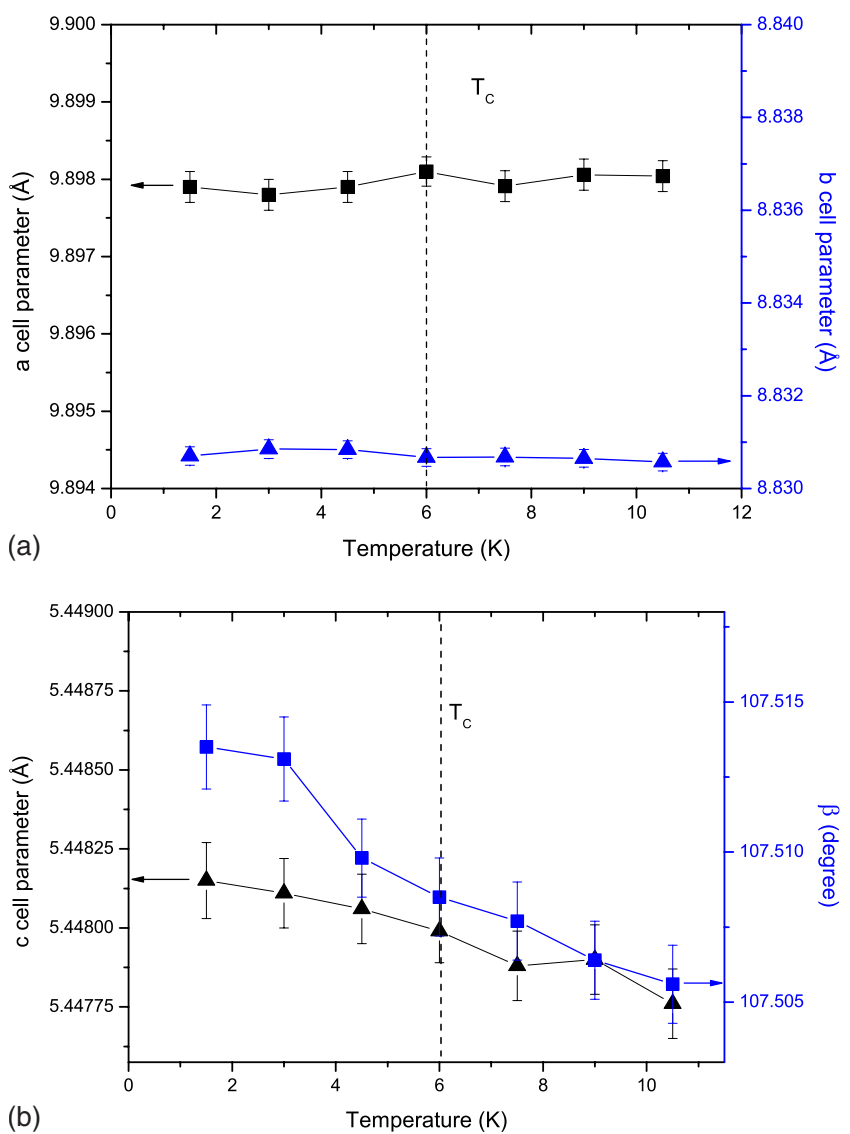

FIG. 5. (Color online) Temperature dependence of (a) cell parameters $a$ (square) and $b$ (triangle) and of the (b) $c$ cell parameter (triangle) and the $\beta$ angle (square) of $\mathrm{NaCrGe}_{2} \mathrm{O}_{6}$ as function of temperature measured with the D1A diffractometer.

$\mu_{e f f}=3.650(2) \mu_{B}$ and an interchain exchange coupling $J^{\prime} / k_{b}=0.200(3) \mathrm{K}$ taking into account $z=4$. The resulting fit is shown together with the experimental data in Fig. 3.

The previous estimation of the interchain coupling was made assuming that the interchain exchange interaction dominates and was found to be $J^{\prime} / k_{b}=0.6 \mathrm{~K} .{ }^{19}$ We believe that this model is more appropriate. Additionally we will see in Sec. III C that the magnetic moment of $\mathrm{NaCrGe}_{2} \mathrm{O}_{6}$ is significantly reduced, which is interpreted as the signature of the low dimension of the magnetic interactions.

TABLE I. Crystallographic coordinates extracted from the Rietveld refinement carried out on powder neutron diffraction (D2B) using the space group $C 2 / c$ at room temperature with $a=9.9114(7) \AA, b$ $=8.8494(6) \AA, c=5.4550(4) \AA$, and $\beta=107.5934(12)^{\circ}$.

\begin{tabular}{llllll}
\hline \hline Atom & Wyckoff & \multicolumn{1}{c}{$x$} & \multicolumn{1}{c}{$y$} & \multicolumn{1}{c}{$z$} & $U_{\text {iso }}$ \\
\hline $\mathrm{Na}$ & $4 e$ & 0 & $0.3013(5)$ & 0.25 & $0.0128(10)$ \\
$\mathrm{Cr}$ & $4 e$ & 0 & $0.9100(5)$ & 0.25 & $0.0065(8)$ \\
$\mathrm{Ge}$ & $8 f$ & $0.29070(13)$ & $0.09652(14)$ & $0.2279(2)$ & $0.0037(3)$ \\
$\mathrm{O}_{1}$ & $8 f$ & $0.10523(15)$ & $0.0803(2)$ & $0.1286(3)$ & $0.0027(3)$ \\
$\mathrm{O}_{2}$ & $8 f$ & $0.35960(19)$ & $0.27448(19)$ & $0.3080(4)$ & $0.0071(4)$ \\
$\mathrm{O}_{3}$ & $8 f$ & $0.3627(2)$ & $0.01175(18)$ & $0.0066(4)$ & $0.0078(4)$ \\
\hline \hline
\end{tabular}


TABLE II. Selected bond distances at room temperature in $\AA$.

\begin{tabular}{llll}
\hline \hline $\mathrm{Cr}^{3+} \mathrm{O}_{6}$ & & \multicolumn{2}{c}{$\mathrm{Ge}^{4+} \mathrm{O}_{4}$} \\
\hline $\mathrm{Cr}^{-} \mathrm{O}_{11}$ & $2.053(4)$ & $\mathrm{Ge}_{1}-\mathrm{O}_{1}$ & $1.7583(19)$ \\
$\mathrm{Cr}^{-} \mathrm{O}_{12}$ & $2.0146(15)$ & $\mathrm{Ge}_{1}-\mathrm{O}_{2}$ & $1.720(2)$ \\
$\mathrm{Cr}^{-} \mathrm{O}_{2}$ & $1.934(3)$ & $\mathrm{Ge}_{1}-\mathrm{O}_{31}$ & $1.747(3)$ \\
& & $\mathrm{Ge}_{1}-\mathrm{O}_{32}$ & $1.756(2)$ \\
\hline \hline
\end{tabular}

\section{B. Structural investigation}

\section{Room-temperature structure}

The refined lattice parameters at room temperature are $a$ $=9.9114(7) \AA, b=8.8494(6) \AA, c=5.4550(4) \AA$, and $\beta$ $=107.5934(12)^{\circ}$. The pattern was refined in the space group $C 2 / c$, taking as starting structural model the atomic coordinates reported for the high-temperature phase of $\mathrm{LiCrSi}_{2} \mathrm{O}_{6}$ (see Ref. 9) and the cell parameters reported by Vasiliev et $a l .{ }^{19}$ The good agreement between the calculated and observed patterns is presented in Fig. 4. The most characteristic parameters after the refinement are listed in Table I. A selection of the most important atomic distances and bonding angles is included in Tables II and III.

We recall here for the general reader the main structural features of the pyroxene family. This system exhibits a chain of edge-sharing $\mathrm{CrO}_{6}$ octahedra running along crystallographic axis $c$. These quasi-one-dimensional chains are connected by chains of $\mathrm{GeO}_{4}$ tetrahedra. The packing of the $\mathrm{CrO}_{6}$ octahedra chains linked by $\mathrm{GeO}_{4}$ tetrahedra chains gives rise to a triangular magnetic lattice.

The $\mathrm{GeO}_{4}$ tetrahedra form infinite chains running parallel to the $c$ axis and are interconnected by the $\mathrm{O}_{3}$ oxygen atoms via shared corners. In the $C 2 / c$ symmetry, there is only one type of $\mathrm{GeO}_{4}$ tetrahedra chain while there are two in the $P 2_{1} / c$ symmetry [see, e.g., $\mathrm{LiCrSi}_{2} \mathrm{O}_{6}$ (Refs. 9, 10, and 14)]. This chain is characterized by the angle $\mathrm{O}_{3}-\mathrm{O}_{3}-\mathrm{O}_{3}$, which is here " $\mathrm{O}$ " rotated. ${ }^{10}$ The $\mathrm{O}_{3}-\mathrm{O}_{3}-\mathrm{O}_{3}$ angle is of $171.0(1)^{\circ}$ at

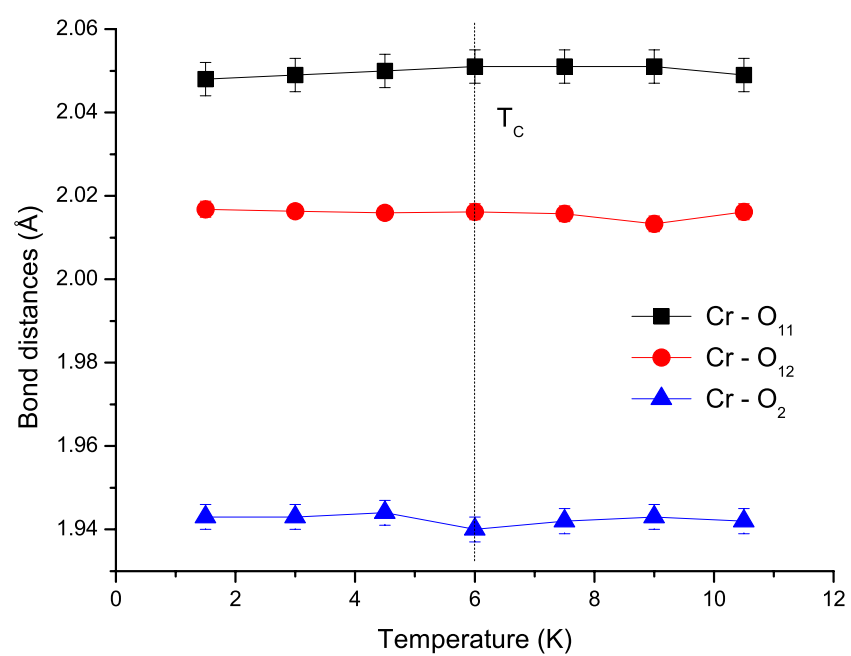

FIG. 6. (Color online) Temperature dependence of the Cr-O bond distances of $\mathrm{NaCrGe}_{2} \mathrm{O}_{6}$ as function of temperature.
TABLE III. Selected bond angles at room temperature in degrees.

\begin{tabular}{lc}
\hline \hline $\mathrm{Cr}_{-} \mathrm{O}_{1}-\mathrm{Cr}$ & $101.88(7)$ \\
$\mathrm{O}_{1}-\mathrm{Cr}-\mathrm{O}_{1}$ & $98.24(6)$ \\
$\mathrm{O}_{2}-\mathrm{Ge}-\mathrm{O}_{1}$ & $116.8(1)$ \\
$\mathrm{O}_{3}-\mathrm{Ge}-\mathrm{O}_{3}$ & $102.7(1)$ \\
\hline \hline
\end{tabular}

room temperature. Our refinement of the room-temperature structure is in good agreement and of similar quality than the recently reported single-crystal structure determination. ${ }^{21}$

\section{Temperature dependence of the nuclear structure}

We have investigated the evolution of the crystal structure through the magnetic ordering temperature using the diffractometer D1A. We plot in Fig. 5 the temperature dependence of the cell parameters below and above the ferromagnetic ordering. As it can be observed, we can hardly notice any anomaly in the cell parameters going through the magnetic transition. This illustrates the absence of a clear magnetostriction within the resolution of our data. Parameter $\beta$ seems to be the most sensitive to this magnetic transition [see Fig. $5(b)]$. This absence of noticeable effect in the structural parameters is further evidenced in the temperature dependence of the $\mathrm{Cr}-\mathrm{O}$ bond distances (see Fig. 6).

While lowering the temperature, the $\mathrm{Ge}-\mathrm{O}_{1}$ and $\mathrm{Ge}-\mathrm{O}_{2}$ bond distances decrease while the $\mathrm{Cr}-\mathrm{O}$ bond distances hardly change (see Figs. 6 and 7). The biggest effect in the structural parameters is observed in the temperature dependence of the $\mathrm{Ge}-\mathrm{O}_{3}$ bond distances. We notice a pronounced differentiation between the two bond distances $\mathrm{Ge}-\mathrm{O}_{31}$ and $\mathrm{Ge}-\mathrm{O}_{32}$ below the critical temperature. Looking at Fig. 8, we observe that the strongest structural effect of the appearance of the ferromagnetic order is localized between the $\mathrm{CrO}_{6}$ octahedra chains. In a first thought, one would expect the strongest magnetostrictive effect to appear along the direction where the magnetic exchange is the larger, e.g., within

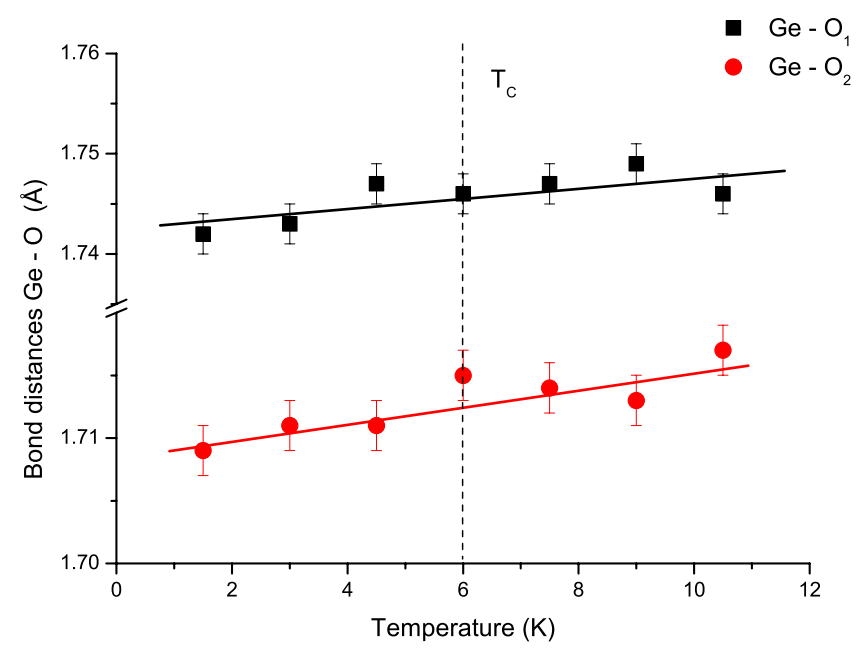

FIG. 7. (Color online) Temperature dependence of the ${\mathrm{Ge}-\mathrm{O}_{1}}$ and $\mathrm{Ge}_{2} \mathrm{O}_{2}$ bond distances of $\mathrm{NaCrGe}_{2} \mathrm{O}_{6}$ as function of temperature. 


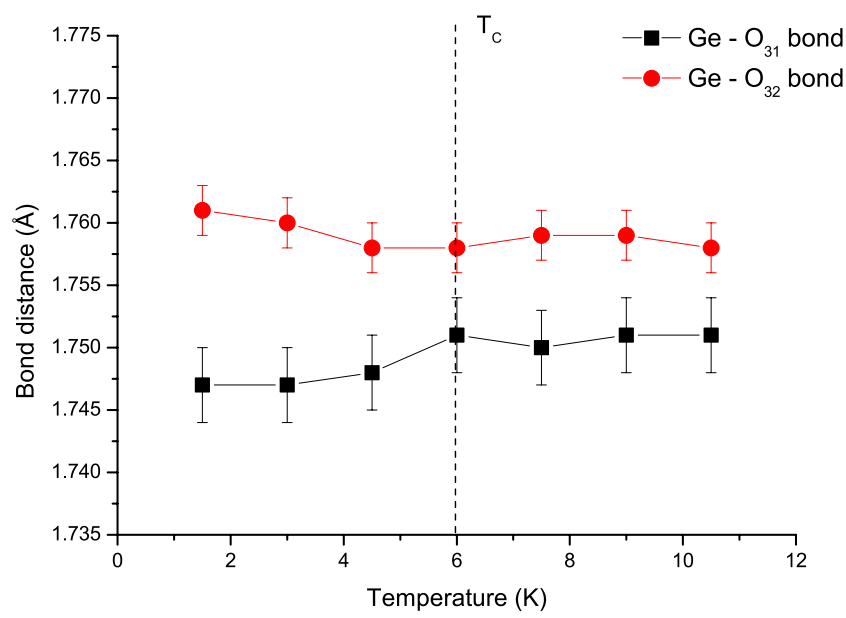

FIG. 8. (Color online) Temperature dependence of the $\mathrm{Ge}-\mathrm{O}_{3}$ bond distances of $\mathrm{NaCrGe}_{2} \mathrm{O}_{6}$ as function of temperature.

the $\mathrm{CrO}_{6}$ octahedra chains. We have to recall here that the $J_{\text {intra }}$ magnetic exchange is about 1 order larger than the $J_{\text {inter }}$ magnetic exchange (see Sec. III A). However, this is due to the existence of a sufficiently strong $J_{\text {inter }}$; that there is occurrence of a 3D long-range magnetic order. Consequently, it is not unreasonable to observe that the biggest magnetoelastic effect occurs between the chain since it is due to the interchain magnetic exchange coupling that we have a 3D magnetic order. Thus we interpret this magnetoelastic effect as the signature of the interchain magnetic exchange coupling constant responsible for the appearance of a long-range order below $6 \mathrm{~K}$.

\section{Determination of the magnetic structure}

The temperature dependence of the neutron powder diffraction patterns collected as function of temperature on the D1B diffractometer is shown in Fig. 9. Below $T \simeq 6 \mathrm{~K}$, one new diffraction peak appears [(110) reflection] with some Bragg peaks increasing in intensity. This confirms the appearance of a magnetic ordering below $T=6 \mathrm{~K}$ in good

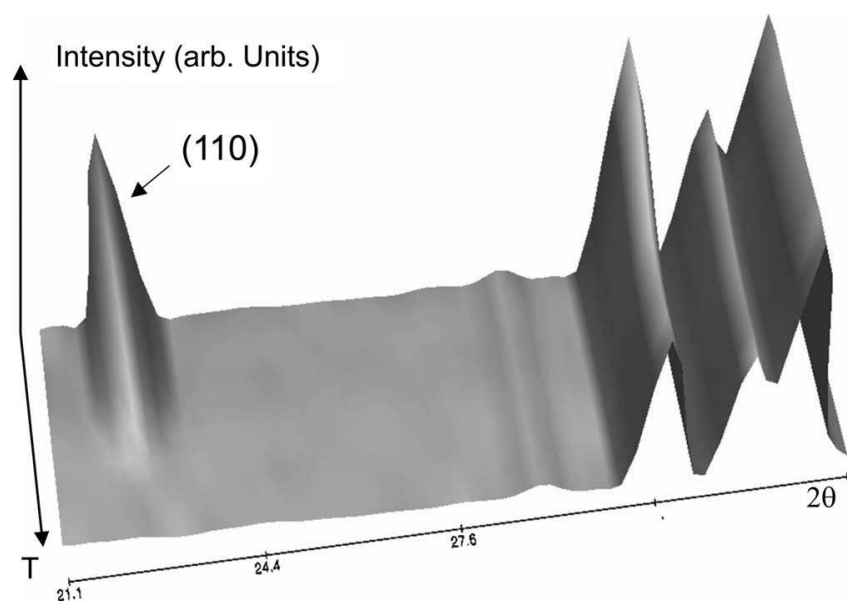

FIG. 9. Neutron-diffraction patterns of $\mathrm{NaCrGe}_{2} \mathrm{O}_{6}$ collected on the $\mathrm{D} 1 \mathrm{~B}$ diffractometer in the temperature range of $1.8-10 \mathrm{~K}$.
TABLE IV. Irreducible representations of the space group $C 2 / c$ for $\mathbf{k}=\mathbf{0}$. The symmetry elements are written according to Kovalev's notation (Ref. 23), $\tau=\left(0,0, \frac{1}{2}\right)$.

\begin{tabular}{lcccc}
\hline \hline & $h_{1}$ & $h_{3} /(\tau)$ & $h_{25} /(\tau)$ & $h_{27} /(\tau)$ \\
\hline$\Gamma_{1}$ & 1 & 1 & 1 & 1 \\
$\Gamma_{2}$ & 1 & 1 & -1 & -1 \\
$\Gamma_{3}$ & 1 & -1 & 1 & -1 \\
$\Gamma_{4}$ & 1 & -1 & -1 & 1 \\
\hline \hline
\end{tabular}

agreement with the magnetic data. ${ }^{19}$ The magnetic reflections can be indexed by the propagation vector $\mathbf{k}=\mathbf{0}$.

The possible magnetic structures compatible with the symmetry of $\mathrm{NaCrGe}_{2} \mathrm{O}_{6}$ are determined by following the representation analysis technique described by Bertaut. ${ }^{22}$ For the propagation vector $\mathbf{k}=\mathbf{0}$, the small group $G_{\mathbf{k}}$, formed by those elements of the space group that leave $\mathbf{k}$ invariant, coincides with the space group $C 2 / c$. For $\mathbf{k}=\mathbf{0}$, the irreducible representations of the group $G_{\mathbf{k}}$ are those shown in Table IV.

A representation $\Gamma$ is constructed with the Fourier components $\mathbf{m}^{\mathbf{k}}$ corresponding to the $\mathrm{Cr}$ atoms of the Wyckoff position $4 e$. The $\mathrm{Cr}$ atoms at the site $4 e$ are denoted as (1) $\left(0, y, \frac{1}{4}\right),(2)\left(\frac{1}{2}, y+\frac{1}{2}, \frac{1}{4}\right),(3)\left(0, \bar{y}, \frac{3}{4}\right)$ and (4) $\left(\frac{1}{2}, \bar{y}+\frac{1}{2}, \frac{3}{4}\right)$. The decomposition of the representation $\Gamma$ in terms of the irreducible representations $\Gamma_{\mathbf{k}}$ is for the $4 e$ site,

$$
\Gamma(4 e)=\Gamma_{1}+\Gamma_{2}+2 \Gamma_{3}+2 \Gamma_{4} .
$$

The different basis vectors [see Eq. (4)] associated with each irreducible representation and calculated by using the projection operator technique implemented in BASIREPS (Ref. 24) are presented in Table V. Despite the fact that there are four magnetic ions, there are only two possible magnetic couplings. Any magnetic moment carried by an ion within the structure is related by the general formula $\vec{S}_{n}$ $=\vec{S}_{k} \exp \left(-i 2 \pi \vec{k} \cdot \overrightarrow{R_{n}}\right)$ where $\overrightarrow{S_{n}}$ is the magnetic moment of the atom considered attached to the lattice point $\overrightarrow{R_{n}}$ for a magnetic wave vector $\vec{k}$. Because of the $C$ centering and having $\vec{k}=\overrightarrow{0}$, the magnetic moment carried by $\mathrm{Cr}_{1}$ is automatically identical to the one carried by $\mathrm{Cr}_{2}$. The same remark holds for the magnetic moments carried by $\mathrm{Cr}_{3}$ and $\mathrm{Cr}_{4}$. Consequently there are as stated above only two possible magnetic couplings,

$$
\begin{aligned}
& \mathbf{M}=\mathbf{S}_{1}+\mathbf{S}_{2}+\mathbf{S}_{3}+\mathbf{S}_{4}, \\
& \mathbf{L}=\mathbf{S}_{1}+\mathbf{S}_{2}-\mathbf{S}_{3}-\mathbf{S}_{4} .
\end{aligned}
$$

TABLE V. Basis vectors for the atoms of the $4 e$ site.

\begin{tabular}{lccc}
\hline \hline Basis vectors & $x$ & $y$ & $z$ \\
\hline$\Gamma_{1}$ & & $M_{y}$ & \\
$\Gamma_{2}$ & & $L_{y}$ & \\
$\Gamma_{3}$ & $M_{x}$ & & $M_{z}$ \\
$\Gamma_{4}$ & $L_{x}$ & & $L_{z}$ \\
\hline \hline
\end{tabular}




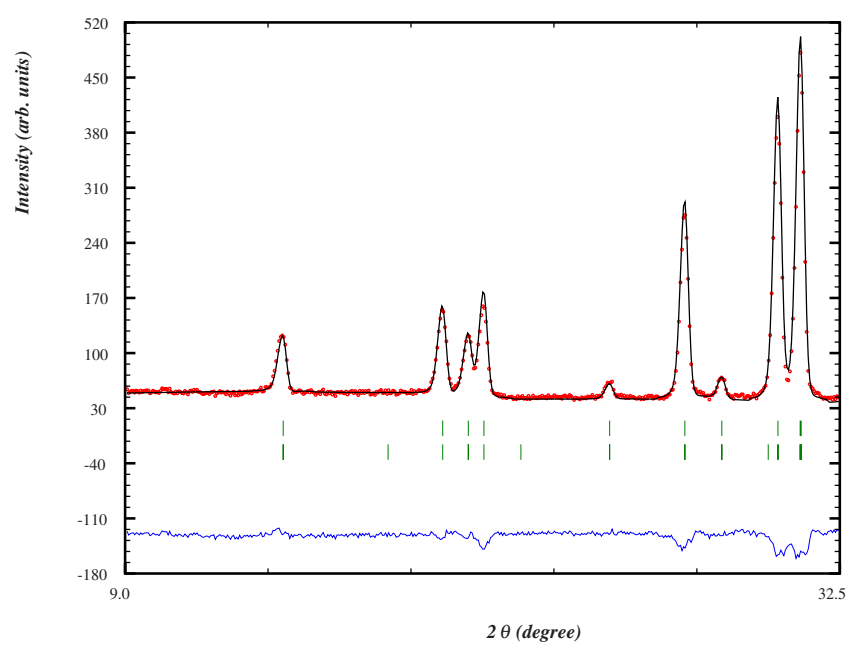

FIG. 10. (Color online) Fragment of the D2B neutron diffraction pattern of $\mathrm{NaCrGe}_{2} \mathrm{O}_{6}$ at $1.7 \mathrm{~K}$ and refined by using the $\Gamma_{3}$ model $\left(\mathrm{Cr}^{3+}\right.$ spins along the $c$ axis, $C 2^{\prime} / c^{\prime}$ symmetry).

According to the SQUID results and taking into account that we have only one single magnetic site, the magnetic structure should be ferromagnetic. Consequently there are only two magnetic structures to be tested: the ones that described the irreducible representations $\Gamma_{1}$ and $\Gamma_{3}$. The best fit using the D2B data is obtained for the magnetic structure described by the irreducible representation $\Gamma_{3}$ (see Fig. 10). Within the experimental resolution, we find that $M_{x}=0$ and $M_{z}=1.85(4) \mu_{B}$ at $1.8 \mathrm{~K}$. This corresponds to a magnetic structure with ferromagnetically coupled chains of $\mathrm{CrO}_{6}$ octahedra.

The thermal evolution of the magnetic moments measured with the D1B diffractometer is displayed in Fig. 11. The value of the magnetic moment approaches saturation only at $2 \mathrm{~K}$. The magnetic moment at $T=1.8 \mathrm{~K}$ is $\left\|\mu\left(\mathrm{Cr}^{3+}\right)\right\|$ $=1.9(2) \mu_{B}$. This is $24(8) \%$ of reduction compared to the saturation of the magnetic form factor of $\mathrm{Cr}^{3+}$ determined experimentally, which is $2.5 \mu_{B} .{ }^{25}$ This magnetic moment is lower by about $9 \%$ compared to $\mathrm{LiCrSi}_{2} \mathrm{O}_{6}$ measured on the same diffractometer. ${ }^{14}$ The reduction in the magnetic moment is larger than in the case of the antiferromagnetic Licontaining pyroxene $\mathrm{Li} M \mathrm{Si}_{2} \mathrm{O}_{6}(M=\mathrm{V}, \mathrm{Cr}, \mathrm{Fe})^{7,11,14} \mathrm{We}$ note that this reduced magnetic moment cannot be explained by covalent effect that would reduce the magnetic moment of $\mathrm{Cr}^{3+}$ ion only by about $5 \%{ }^{26}$

For an explanation of the reduced magnetic moment, one can consider two possibilities: low-dimensional system and/or magnetic frustration. While $\mathrm{NaCrGe}_{2} \mathrm{O}_{6}$ is a good representative of a magnetic triangular lattice, for ferromagnetic interactions, this network cannot give rise to magnetic frustration. Consequently the other possibility is to consider that the reduction in the magnetic moment can arise from the low dimensionality of the system. The distance $\mathrm{Cr}-\mathrm{Cr}$ within the chains is 3.159(3) $\AA$ while the distance between the chains is $5.45527(5) \AA$. Thus in a first approximation $\mathrm{NaCrGe}_{2} \mathrm{O}_{6}$ can be considered as 1D ferromagnet. The low dimensionality of the magnetic lattice for Heisenberg system can give

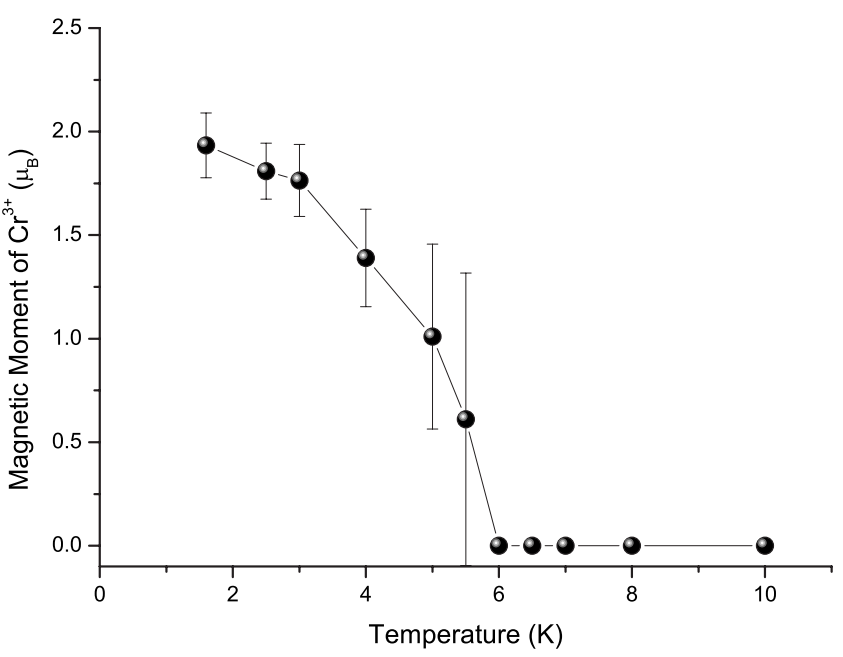

FIG. 11. Neutron-diffraction patterns collected on the D1B diffractometer in the temperature range $1.8-10 \mathrm{~K}$.

rise to a significant reduction in the magnetic moment. This has been already observed in the 1D Heisenberg antiferromagnet $\mathrm{NiSb}_{2} \mathrm{O}_{6}$. In this system, the magnetic moment at 1.5 $\mathrm{K}$ is reduced by $20 \%$, which is comparable to our system. ${ }^{27}$ Consequently, we interpret the reduction in the magnetic moment of $\mathrm{NaCrGe}_{2} \mathrm{O}_{6}$ as the signature of the low dimensionality of the system.

\section{CONCLUSION}

We have investigated the magnetic and crystal structures of $\mathrm{NaCrGe}_{2} \mathrm{O}_{6}$ as function of temperature using SQUID magnetometry and neutron powder diffraction. Jodlauk et $a l .{ }^{5}$ suggested that several members of the pyroxene family should exhibit a spiral magnetic structure resulting from the magnetic frustration. This would explain the reported multiferroic properties. Our experimental results show that $\mathrm{NaCrGe}_{2} \mathrm{O}_{6}$ exhibits a ferromagnetic ordering below $T_{C}$ $=6 \mathrm{~K}$. The magnetic structure is commensurate with the chemical unit cell with $\mathbf{k}=\mathbf{0}$ with a magnetic moment of $\mathrm{Cr}=1.85(4) \mu_{B}$ at $1.8 \mathrm{~K}$. The magnetic space group displayed by $\mathrm{NaCrGe}_{2} \mathrm{O}_{6}$ is $C 2^{\prime} / c^{\prime}$. This magnetic symmetry does not allow a linear magnetoelectric effect. ${ }^{28}$ No structural phase transition was observed down to $1.8 \mathrm{~K}$. $\mathrm{NaCrGe}_{2} \mathrm{O}_{6}$ shows a significant reduction in its magnetic moment by $24(8) \%$, which results from the low dimensionality of the system. We correlate the structural changes between the chains as the structural signature of the existence of a sufficiently strong magnetic exchange coupling, which is responsible for the appearance of the ferromagnetic state below $T_{C}=6 \mathrm{~K}$.

\section{ACKNOWLEDGMENTS}

This study is partly supported by the Ministry of Education, Culture, Sports, Science and Technology of Japan under Grant in Aid for Scientific Research No. 19052008, RFBR under Grant No. 07-02-91201, and JSPS (Japan)-RFBR (Russia) joint research project. 
*Corresponding author; nenert@ill.eu

${ }^{1}$ M. Fiebig, J. Phys. D 38, R123 (2005).

${ }^{2}$ W. Eerenstein, N. D. Mathur, and J. F. Scott, Nature (London) 442, 759 (2006).

${ }^{3}$ S.-W. Cheong and M. Mostovoy, Nature Mater. 6, 13 (2007).

${ }^{4}$ A. Pimenov, A. A. Mukhin, V. Yu. Ivanov, V. D. Travkin, A. M. Balbashov, and A. Loidl, Nat. Phys. 2, 97 (2006); A. B. Sushkov, R. V. Aguilar, S. Park, S.-W. Cheong, and H. D. Drew, Phys. Rev. Lett. 98, 027202 (2007).

${ }^{5}$ S. Jodlauk, P. Becker, J. A. Mydosh, D. I. Khomskii, T. Lorenz, S. V. Streltsov, D. C. Hezel, and L. Bohatý, J. Phys.: Condens. Matter 19, 432201 (2007).

${ }^{6}$ M. Isobe, E. Ninomiya, A. N. Vasiliev, and Y. Ueda, J. Phys. Soc. Jpn. 71, 1423 (2002); S. V. Streltsov, O. A. Popova, and D. I. Khomskii, Phys. Rev. Lett. 96, 249701 (2006).

${ }^{7}$ M. D. Lumsden, G. E. Granroth, D. Mandrus, S. E. Nagler, J. R. Thompson, J. P. Castellan, and B. D. Gaulin, Phys. Rev. B 62, R9244 (2000).

${ }^{8}$ M. Isobe and Y. Ueda, J. Magn. Magn. Mater. 272-276, 948 (2004); S. V. Streltsov and D. I. Khomskii, Phys. Rev. B 77, 064405 (2008); G. J. Redhammer, H. Ohashi, and G. Roth, Acta Crystallogr., Sect. B: Struct. Sci. B59, 730 (2003); C. Satto, P. Millet, and J. Galy, Acta Crystallogr., Sect. C: Cryst. Struct. Commun. 53, 1727 (1997); P. Millet, F. Mila, F. C. Zhang, M. Mambrini, A. B. Van Oosten, V. A. Pashchenko, A. Sulpice, and A. Stepanov, Phys. Rev. Lett. 83, 04176 (1999); E. Baum, W. Treutmann, M. Behruzi, W. Lottermoser, and G. Amthauer, Z. Kristallogr. 183, 273 (1988); B. Pedrini, S. Wessel, J. L. Gavilano, H. R. Ott, S. M. Kazakov, and J. Karpinski, Eur. Phys. J. B 55, 219 (2007); M. Isobe, Y. Ueda, A. N. Vasiliev, T. N. Voloshok, and O. L. Ignatchik, J. Magn. Magn. Mater. 258-59, 125 (2003); A. N. Vasiliev, O. L. Ignatchik, A. N. Sokolov, Z. Hiroi, M. Isobe, and Y. Ueda, JETP Lett. 78, 551 (2003).

${ }^{9}$ G. J. Redhammer, and G. Roth, Z. Kristallogr. 219, 585 (2004). Please note that the coordinates given in the paper contain an error. The $x$ coordinate of $\mathrm{Si}_{1}$ is 0.0487 and not 0.487 .

${ }^{10}$ G. J. Redhammer and G. Roth, Z. Kristallogr. 219, 278 (2004).

${ }^{11}$ G. J. Redhammer, G. Roth, W. Paulus, G. André, W. Lottermoser, G. Amthauer, W. Treutmann, and B. KoppelhuberBitschnau, Phys. Chem. Miner. 28, 337 (2001).

${ }^{12}$ W. Lottermoser, G. J. Redhammer, K. Forcher, G. Amthauer, W.
Paulus, G. André, and W. Treutmann, Z. Kristallogr. 213, 101 (1998).

${ }^{13}$ O. Ballet, J. M. D. Coey, G. Fillion, A. Ghose, A. Hewat, and J. R. Regnard, Phys. Chem. Miner. 16, 672 (1989).

${ }^{14}$ G. Nénert, M. Isobe, C. Ritter, O. Isnard, A. N. Vasiliev, and Y. Ueda, Phys. Rev. B 79, 064416 (2009).

${ }^{15}$ G. Nénert, M. Isobe, C. Ritter, A. N. Vasiliev, and Y. Ueda (unpublished).

${ }^{16}$ Y. Matsushita, F. Izumi, M. Isobe, and Y. Ueda, Solid State Sci. (to be published 2009).

${ }^{17}$ W.-M. Zhang, W. M. Saslow, and M. Gabay, Phys. Rev. B 44, 5129 (1991).

${ }^{18}$ J. Rodríguez-Carvajal, Physica B 192, 55 (1993).

${ }^{19}$ A. N. Vasiliev, O. L. Ignatchik, A. N. Sokolov, Z. Hiroi, M. Isobe, and Y. Ueda, Phys. Rev. B 72, 012412 (2005).

${ }^{20}$ J. C. Bonner and M. E. Fisher, Phys. Rev. 135, A640 (1964); T. Smith and S. A. Friedberg, ibid. 176, 660 (1968).

${ }^{21}$ G. J. Redhammer, G. Roth, and G. Amthauer, Acta Crystallogr., Sect. C: Cryst. Struct. Commun. 64, i97 (2008).

${ }^{22}$ E. F. Bertaut, in Magnetism, edited by G. T. Rado and H. Shul (Academic, New York, 1963), Vol. III, Chap. 4.

${ }^{23}$ O. V. Kovalev, in Representations of the Crystallographic Space Groups: Irreducible Representations, Induced Representations and Corepresentations, edited by H. T. Stokes and D. M. Hatch (Gordon and Breach, Amsterdam, 1993).

${ }^{24}$ J. Rodríguez-Carvajal, BASIREPS: a program for calculating irreducible representations of space groups and basis functions for axial and polar vector properties (see http://wwwold.ill.fr/dif/ Soft/fp/php/downloads.html).

${ }^{25}$ P. J. Brown, J. B. Forsyth, and F. Tasset, Solid State Sci. 7, 682 (2005).

${ }^{26}$ R. Nathans, H. A. Alperin, S. J. Pickart, and P. J. Brown, J. Appl. Phys. 34, 1182 (1963).

${ }^{27}$ E. Ramos, F. Fernández, A. Jerez, C. Pico, J. RodríguezCarvajal, R. Saez-Puche, and M. L. Veiga, Mater. Res. Bull. 27, 1041 (1992); H. Ehrenberg, G. Wltschek, and J. RodríguezCarvajal, J. Magn. Magn. Mater. 184, 111 (1997).

${ }^{28}$ Physical Properties of crystals, International Tables for Crystallography, edited by A. Authier (Kluwer Academic Publishers, New York, 2003), Vol. D. 